\title{
Design of a Hybrid Energy-Harvesting Shock Absorber
}

\author{
Longhan $\mathrm{Xie}^{\mathrm{a}}$, Jiehong $\mathrm{Li}^{\mathrm{b}}$ and Mingjing $\mathrm{Cai}^{\mathrm{c}}$ \\ School of Mechanical and Automotive Engineering, South China University of Technology, \\ Guangzhou, China \\ amelhxie@scut.edu.cn, ${ }^{\mathrm{b}} 406607871 @ q q . c o m,{ }^{\mathrm{c}}$ calls.cai@163.com
}

KEYWORDS: Hybrid Energy-Harvesting, 3D modeling, conventional damper

Abstract. This work is to study a novel hybrid energy-harvesting shock absorber for vehicle to provide the required damping function to reduce the vibration between vehicle and roads, as well as to reclaim vibration energy from automotive suspension system during driving. The hybrid energy-harvesting shock absorber consists of the traditional hydraulic damping system and the energy harvesting system to convert the mechanical energy into electricity. The hybrid shock absorber was design, and analysis was conducted. Simulation shows that the hybrid energy-harvesting shock absorber can regenerate considerable electricity.

\section{Introduction}

Suspension is one of the most important parts of the vehicle, which connects the wheels to the car body. Due to the roughness of the road, hydraulic shock absorber is used to absorb the vibration. Although this way can overcome the vibration rapidly, but the vibration energy is inevitably wasted. In order to extract the vibration energy to drive the vehicle, the regenerative shock absorber has been suggested. During the past decades, many researchers explored different principles and designs of regenerative shock absorber. Wang[1] et al proposed an idea of using the hydraulic motor to drive an electric generator in order to generate electric. Wendel[2] used a regenerative damp to extract the vibration energy of suspension. Noritsugu[3] investigated a pneumatic drive system, which store a part of the exhaust gas in order to reuse it in the air tank. Li and Tse[4]developed an energy-harvesting hydraulic damper to extract the vibration energy from suspension. Chen and Liao [5], as well as Sapinski [6]proposed to use MR damper to dismiss the vibration and extract the vibration energy. Linear electromagnetic generator [7], rack-pinion[8] and ball-screw [9] were also used to construct the energy harvesting shock to harness the vibration.

This paper aims at design and simulation of a hybrid energy-harvesting shock absorber, which combines a traditional hydraulic absorber and an electromagnetic energy harvesting mechanism so that the shock absorber can quickly absorb the vibration and convert it to electricity. This paper is organized as follow. In second section, the working principle of the energy-harvesting shock is introduced. In third section, the design and 3D modeling of the shock absorber is given; and in the fourth section, analysis and simulation of the electromagnetic energy harvesting shock is conducted. Then the conclusion is given in last section.

\section{The working principle}

The schematic of the hybrid energy-harvesting shock absorber is shown as Fig.1. The novel hybrid shock absorber is similar to the traditional hydraulic damper. There are two hoops designed to connect the suspension system and the tire. The hybrid shock absorber consists of two subsystem: one is the hydraulic damping system, and the other is the energy harvesting system. The hydraulic damping system consists of a main spring, a main piston, a cylinder and two secondary pistons. When the main piston move up and down, it drives the hydraulic oil and convert the vibration energy to hydraulic energy; and due to the viscosity of hydraulic oil, vibration of the car body can be released. The energy harvesting system consists of secondary pistons, rack gears, gear train and generators. The secondary piston connects with the rack gear, and when the main piston drives the liquid in the cylinder container, the hydraulic pressure will drive the secondary piston to move up and down. And then, the rack gear 
will follow the secondary piston, who will go up and down to drive the gear train. The gear train is used to accelerate the movement, which will finally rotate the generator to produce electricity. Due to the random vibration between the vehicle and roads, the generated electricity needs to be rectified through a regulating circuit and then to be stored in a battery for use. And thus, the hydraulic damping system satisfies the function request of automotive shock absorber, and the energy harvesting system is added to shock absorber to reclaim the vibration energy and convert it to electricity.

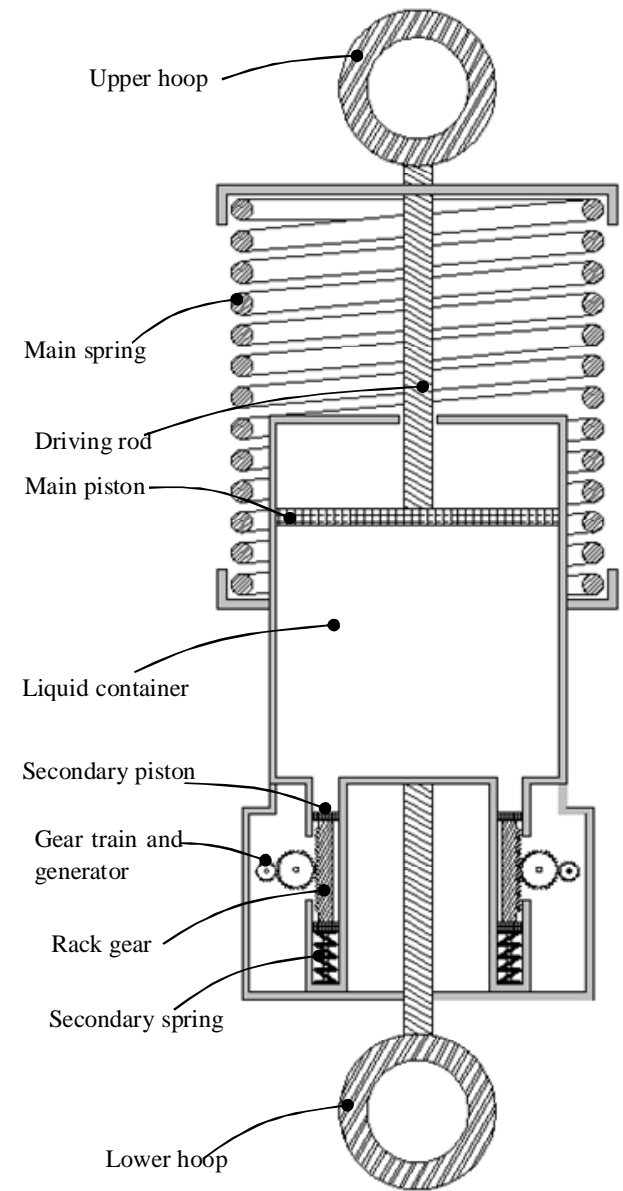

Fig.1: Schematic of the hybrid energy-harvesting shock (a)

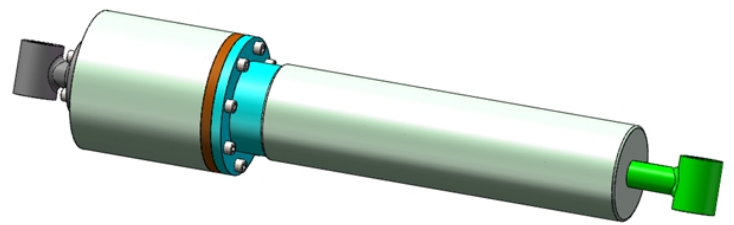

(b)

(c)

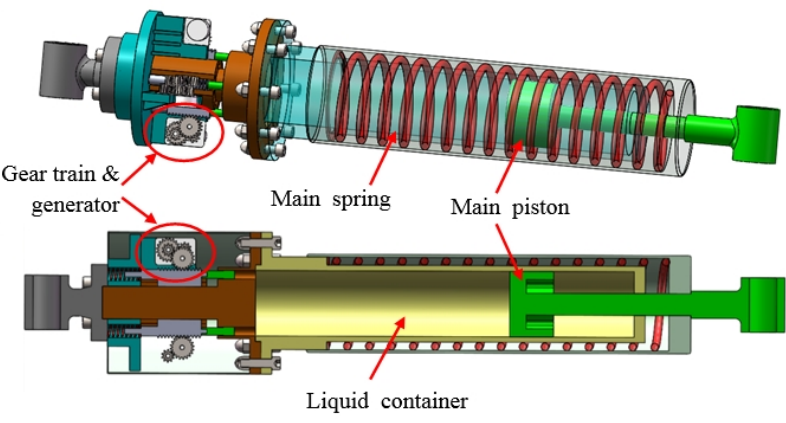

Fig.2: the 3D model of the hybrid shock absorber

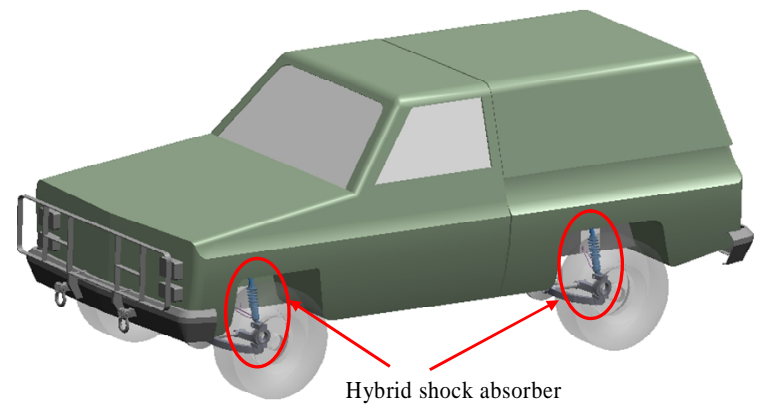

Fig. 3: The hybrid shock absorber in the vehicle

\section{The 3D model}

Following the working principle in Fig.1, a model of prototype was developed. The 3D model and its design detail are given in Fig.2. Fig.2(a) shows the overview of the hybrid energy-harvesting shock absorber, which has a similar configuration and sizes with traditional hydraulic shock absorber, and which is packaged in a cylinder-like body. The hybrid shock absorber provides two hoops to assembly with the vehicle suspension system and tire. In addition, the configuration of energy harvester system is compact, so it is also easy to mount on the car body. Fig.2(b) illustrates the design detail of the hybrid shock absorber and Fig.2(c) gives the axially sectional view. There are two sets of energy harvesting subsystems in the prototype of the hybrid shock absorber, and actually, it can be installed more subsystems according to actual requirements.

The hybrid energy-harvesting shock absorber can be fit for regular vehicle. Figure 3 shows the assembly of the hybrid shock absorber mounting on a vehicle, which has the same assembly method with traditional hydraulic damper. 


\section{Analysis and Simulation}

When vehicles travel on the road, it will receive excitation from road roughness, baking forces etc., which discomfort the drivers. Shock absorbers can reduce the vibration by dissipating the vibration energy into heat waste. Although conventional damper can reduce the vibration effectively, its damping force is a constant. The conventional damper can be modelled as Fig.4. $\mathrm{m}_{1}$ represents the unsprung mass and $\mathrm{m}_{2}$ represents sprung mass. The input of the system is the road roughness $\mathrm{y}_{0}$. This system has two degree of freedom, the movement of unsprung mass $\mathrm{y}_{1}$ and the movement of sprung mass $\mathrm{y}_{2}$. In addition, $\mathrm{k}_{1}$ is stiffness of the tire and $\mathrm{k}_{2}$ is stiffness of the suspension's spring. Moreover, $\mathrm{C}_{1}$ is a damping coefficient between the tire and the road, and $\mathrm{C}_{1}$ is considered to be a damping coefficient of the shock absorber. With the energy harvesting system in the shock absorber, the damper converts the vibration energy into electric energy, and it's damping coefficient is $\mathrm{C}_{2}$, which is changing according to the vibration between the vehicle and roads. After the electricity is rectified by electric circuit, it can be stored in the battery.

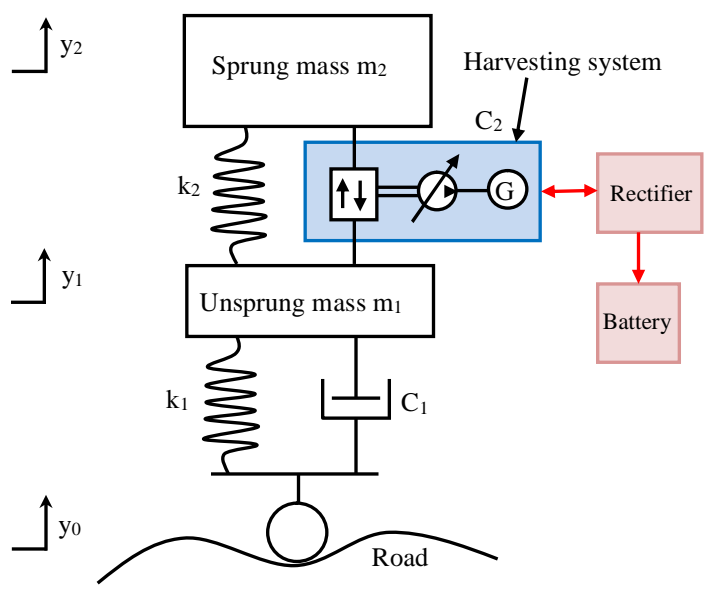

Fig. 4: The model of hybrid energy harvesting shock absorber with a quarter car model

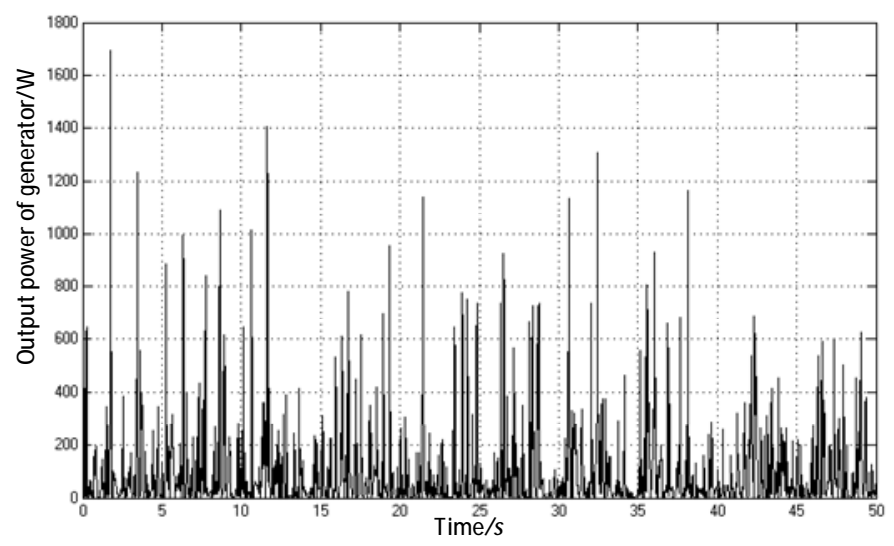

Fig.5: The output power of the generator under the vehicle velocity at $64 \mathrm{~km} / \mathrm{h}$ of the road with surface of Rank C

Based on the two-degree-freedom system of Fig.4, the governing equation can be obtain as following:

$$
\left\{\begin{array}{l}
m_{2} y_{2}+C_{2}\left(y_{2}-y_{1}\right)+k_{2}\left(y_{2}-y_{1}\right)=0 \\
m_{1} y_{1}+C_{1}\left(y_{1}-y_{0}\right)+k_{1}\left(y_{1}-y_{0}\right)-C_{2}\left(y_{2}-y_{1}\right)-k_{2}\left(y_{2}-y_{1}\right)=0
\end{array}\right.
$$

where the coefficients including of $\mathrm{m}_{1}, \mathrm{~m}_{2}, \mathrm{C}_{1}, \mathrm{k}_{1}$ and $\mathrm{k}_{2}$ are constant, only $\mathrm{C}_{2}$ is a variable. The coefficient $\mathrm{y}_{0}$ is the input of the system.

In oder to analyze the power output of the hybrid energy-harvesting shock absorber, we adopt a white noise as excitation bewteen the vehicle and roads $=-2 \pi f_{0} y_{0}+2 \pi \sqrt{G_{0} U_{0}} w$, where $\mathrm{y}_{0}$ is the excitation of the road, $\mathrm{G}_{0}$ is the coefficient of the road surface roughness, $\mathrm{U}_{0}$ is the velocity of vehicle, $\mathrm{w}$ is the Gaussian white noise with the mean value of zero, and $\mathrm{f}_{0}$ is lower cutoff frequency with the range from 0.06 to $0.07 \mathrm{~Hz}$. The Fig. 5 shows the output power under the vehicle velocity at $64 \mathrm{~km} / \mathrm{h}$ of the road with surface of Rank C. Under different vehicle velocity and different road conditions, the average power output from the generator is given in Fig.6. So we can find that the worse condition of road, the more power produced. 


\section{Conclusions}

This work is to design and manufacture a novel hybrid energy-harvesting shock absorber with energy harvesting to reclaim vibration energy from automotive suspension system during driving. The hybrid shock absorber consists of the traditional hydraulic damping system to absorb the vibration between the vehicle and roads, and the energy harvesting system to convert the mechanical energy into electricity. The 3D model of the hybrid energy-harvesting shock absorber was built, and analysis was carried out. Analysis shows that the hybrid energy harvesting shock absorber can produce considerable electricity during vehicle in motion. The prototype and experiments will be carried out in future work.

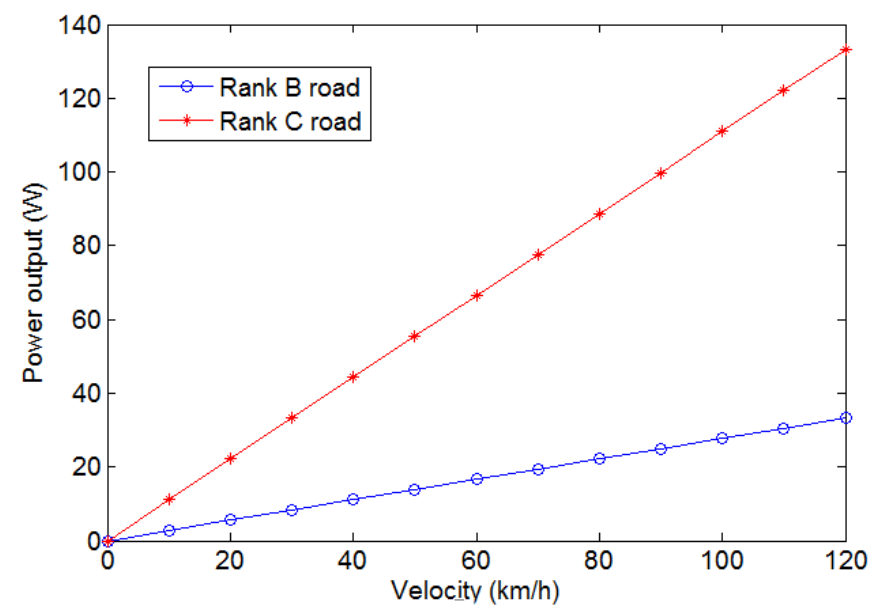

Fig. 6: The output power of different velocity and different road condition

\section{Acknowledgments}

This work was supported by the Fundamental Research Funds for the Central Universities (Grant No. 2014ZZ0020) and the Industrial Technology Research and Development Funds of Guangdong Province (Grant No. 2013B09060).

\section{References}

[1]. Ruichen Wang, Zhi Chen, Haijun Xu, Karsten Schmidt, Fengshou Gu and Andrew.D.Ball. Modelling and Validation of a Regenerative Shock Absorber System. Proceedings of the 20th International Conference on Automation \& Computing, Cranfield University [C]. Bedfordshire, UK, 12-13 September 2014

[2]. Fodor M, Redfield R. The variable linear transmission for regenerative damp ing in vehicle suspension control [ J ]. Vehicle System Dynamics, 1993, 22 (1) : 1 20.

[3]. Noritsugu T. Energy saving of a pneumatic system (2). Energy regenerative control of a pneumatic drive system. Application to active air suspension [J]. Hydraulics \& Pneumatics, 1999, 38 (4) : $1 \sim 4$.

[4]. Chuan Li and Peter W Tse. Fabrication and testing of an energy-harvesting hydraulic damper[J]. Smart Mater. Struct. 2013

[5]. ChaoChen, Wei-Hsin Liao. A self-sensing magnetorheological damper with power generation[J]. Smart Mater. Struct, 2012

[6]. Bogdan Sapinski. Energy-harvesting linear MR damper: prototyping and testing[J]. Smart Mater.Struct, 2014

[7]. Lei Zuo, Design and characterization of an electromagnetic energy harvester for vehicle suspensions[J]. Smart Materials and Structures, 2010, 19

[8]. Weeks D. A., Beno J. H., Guenin A. M., Bresie D. A.. Electromechanical Active Suspension 
Demonstration for Off-Road Vehicles[C]. SAE, 2000.

[9]. Ryuzo Hayashi, Yoshihiro Suda, Kimihiko Nakano. Anti-Rolling Suspension for an Automobile by Coupled Electromagnetic Devices [J]. Journal of Mechanical Systems for Transportation and Logistics, 2008, Vol.(1): 43-55. 